PROCEEDINGS OF THE

AMERICAN MATHEMATICAL SOCIETY

Volume 132, Number 4, Pages 1113-1123

S 0002-9939(03)07194-6

Article electronically published on October 9, 2003

\title{
SPECTRAL RADII AND EIGENVALUES OF SUBDIVISION OPERATORS
}

\author{
DI-RONG CHEN
}

(Communicated by David R. Larson)

\begin{abstract}
This paper discusses the spectra of matrix subdivision operators. We establish some formulas for spectral radii of subdivision operators on various invariant subspaces in $\ell_{p}$. A formula for the spectral radius of a subdivision operator, in terms of the moduli of eigenvalues, is derived under a mild condition. The results are even new in the scalar case. In this case, we show that the subdivision operator has no eigenvector in $\ell_{p}$ if the corresponding subdivision scheme converges for some $p \in[1, \infty)$.
\end{abstract}

\section{$\S 1$. INTRODUCTION}

This paper establishes some formulas for the spectral radii of subdivision operators with a matrix mask. Some relations among the spectral radii of the subdivision operator, the moduli of eigenvalues of the subdivision operator, and joint spectral radii of two finite matrices are discussed. Using a formula for the spectral radii, we prove that, in some cases, there exists no eigenvector of subdivision operators in $\ell_{p}$.

Let $\mathbb{C}^{r \times m}$ be the space of $r \times m$ matrices with entries being complex numbers. Denote by $(\ell(\mathbb{Z}))^{r \times m}$ the set of all sequences $\lambda=(\lambda(\alpha))_{\alpha \in \mathbb{Z}}, \lambda(\alpha) \in \mathbb{C}^{r \times m}$. Suppose that $N$ is a positive integer and that $a \in(\ell(\mathbb{Z}))^{r \times r}$ satisfying $a(\alpha)=0 \forall \alpha \notin$ $\{0,1, \ldots, N\}$. The (matrix) subdivision operator $S_{a}$, associated with mask $a$, is an operator defined on the space $\left(\ell\left(\mathbb{Z}^{s}\right)\right)^{l \times r}$ as follows:

$$
S_{a} c(\alpha):=\sum_{\beta \in \mathbb{Z}^{s}} c(\beta) a(\alpha-2 \beta), \quad \alpha \in \mathbb{Z}^{s} .
$$

The subdivision scheme with mask a generates control points $c^{n}=\left(c^{n}(\alpha)\right)_{\alpha \in \mathbb{Z}}$ at dyadic points $\alpha / 2^{n}, \alpha \in \mathbb{Z}$, recursively by

$$
c^{n}=S_{a} c^{n-1}, \quad n=1,2, \ldots, \text { and } c^{0}=c .
$$

Before moving further we need some notation. Assume that $\mathbb{C}^{r \times m}$ is equipped with a norm $\|\cdot\|$. Let $\left(\ell_{p}(\mathbb{Z})\right)^{r \times m}$ be the normed linear space of sequences $\lambda=$

Received by the editors February 21, 2001 and, in revised form, December 12, 2002.

2000 Mathematics Subject Classification. Primary 42C15, 47B06.

Key words and phrases. Subdivision operator, spectral radius, joint spectral radius.

Supported in part by NSF of China under Grant 10171007 and City University of Hong Kong under Grant 7001442.

(C)2003 American Mathematical Society 
$(\lambda(\alpha))_{\alpha \in \mathbb{Z}}$ such that $\|\lambda\|_{p}<\infty$, where

$$
\|\lambda\|_{p}:=\left(\sum_{\alpha \in \mathbb{Z}}\|\lambda(\alpha)\|^{p}\right)^{1 / p}, \quad 1 \leq p<\infty,
$$

and $\|\lambda\|_{\infty}:=\sup _{\alpha \in \mathbb{Z}}\|\lambda(\alpha)\|$. Clearly, the set $\left(\ell_{p}(\mathbb{Z})\right)^{r \times m}$ is independent of the choice of norm $\|\cdot\|$ on $\mathbb{C}^{r \times m}$, and any two norms on $\mathbb{C}^{r \times m}$ induce two equivalent norms on $\left(\ell_{p}(\mathbb{Z})\right)^{r \times m}$. In what follows, no attention will be paid to the notation of norms on $\mathbb{C}^{r \times r}$.

Let $W_{p}(\mathbb{R})$ denote $L_{p}(\mathbb{R})$ for $1 \leq p<\infty$ and the space $C_{u}(\mathbb{R})$ of uniformly continuous and bounded functions on $\mathbb{R}$ for $p=\infty$. $W_{p}(\mathbb{R})$ is a Banach space with the usual norm $\|\cdot\|_{p}$.

We are ready to define the convergence of a matrix subdivision scheme. Following 10] and 11], we say that the subdivision scheme, associated with mask a, converges in $L_{p}$ if, for any $c \in\left(\ell_{p}(\mathbb{Z})\right)^{l \times r}$, there is a matrix-valued function $f_{c} \in\left(W_{p}(\mathbb{R})\right)^{l \times r}$ such that

$$
\lim _{n \rightarrow \infty} 2^{-n / p}|| S_{a}^{n} c-\mu_{p}^{n}\left(f_{c}\right) \|_{p}=0,
$$

and $f_{c} \neq 0$ for some $c \in\left(\ell_{p}(\mathbb{Z})\right)^{l \times r}$, where $\mu_{p}^{n}(g) \in\left(\ell_{p}(\mathbb{Z})\right)^{l \times r}$, for a matrix-valued function $g \in\left(W_{p}(\mathbb{R})\right)^{l \times r}$ is given by

$$
\mu_{p}^{n}(g)=\left(2^{n} \int_{\alpha / 2^{n}}^{(\alpha+1) / 2^{n}} g(x) d x\right)_{\alpha \in \mathbb{Z}^{\prime}}, \quad 1 \leq p<\infty
$$

and $\mu_{\infty}^{n}(g)=\left(g\left(\alpha / 2^{n}\right)\right)_{\alpha \in \mathbb{Z}}$.

The vector subdivision schemes studied by [10] and [11, etc. correspond to the case $l=1$. We note that either the convergence of the subdivision scheme $S_{a}^{n}$ or the spectral radius $\rho_{p}\left(S_{a}\right)$ of $S_{a}$ on $\left(\ell_{p}(\mathbb{Z})\right)^{l \times r}$ is independent of $l$. Nevertheless we find it convenient sometimes to consider $S_{a}$ as an operator on $\left(\ell_{p}(\mathbb{Z})\right)^{l \times r}$ for general $l$ instead of $l=1$.

Subdivision schemes with matrix mask are a natural extension of subdivision schemes with scalar mask. They play an important role in computer graphics and wavelet analysis. The convergence of subdivision schemes has been studied extensively. There is a direct connection between the spectral radius of a subdivision operator $S_{a}$ on the one hand, and the convergence of a subdivision scheme $S_{a}^{n}$ on the other hand ([1], [10], [11], etc). When $p$ is an even integer, $\rho_{p}\left(S_{a}\right)$ is realizable as the largest eigenvalue (in modulus) of an associated operator defined on a finitedimensional space [14]. As for the spectrum of $S_{a}$, it was proved in [15] that for any $1 \leq p \leq \infty$, except for finitely many eigenvalues of $S_{a}$, any number in $\left\{z \in \mathbb{C}:|z|<\rho_{p}\left(S_{a}\right)\right\}$ lies in the residual spectrum of $S_{a}$.

It is known that the spectral radius of a subdivision operator may be expressed in terms of the $p$-joint spectral radius. An equality between the spectral radius $\rho_{\infty}\left(S_{a}\right)$ and the uniform joint spectral radius introduced in [12 was established in [5]. By use of the notion of $p$-joint spectral radius, Jia [7] extended this result to the general case $1 \leq p \leq \infty$.

In this paper, we are particularly interested in the following formula for the spectral radius $S_{a}$ established for $l=r=1$ in [5]:

$$
\rho_{p}\left(S_{a}\right)=\lim _{n \rightarrow \infty}\left\|S_{a}^{n} \delta\right\|_{p}
$$


where $\delta=(\delta(\alpha))_{\alpha \in \mathbb{Z}} \in \ell(\mathbb{Z})$ is given by $\delta(0)=1$ and $\delta(\alpha)=0 \forall \alpha \neq 0$. One of the goals of this paper is to extend equality (1.2) by replacing $\delta$ with any $c \in$ $\left(\ell_{p}(\mathbb{Z})\right)^{l \times r}$ satisfying a mild condition. The flexibility of $c$ is used for the study of the spectral properties of $S_{a}$. For example, we prove easily that the largest modulus of $l$ eigenvalues is equal to $\rho_{p}\left(S_{a}\right)$ provided that the corresponding eigenvectors satisfy that mild condition (see Theorem 2.7). As one of its corollaries, Theorem 3.1 generalizes a result proved by [6] to the case $1 \leq p \leq 2$. An application of the results to the studies of regularity of refinable vectors and the convergence of vector cascade algorithms is given in [3].

It is worth noting that the spectrum of the subdivision operator $S_{a}$, as an operator on $(\ell(\mathbb{Z}))^{l \times r}$, is also related with some properties of the corresponding refinable vector. Under a weak condition, the approximation order of the refinable vector can be characterized in terms of the eigenvectors of polynomial sequences (see [ and [2], etc).

The paper is organized as follows. The notion of $p$-joint spectral radius will be given in the remaining part of this section. In Section 2, some formulas for the spectral radii of $S_{a}$ on various invariant subspaces are given in terms of both $p$ joint spectral radii and some limits. As a corollary, under a mild condition on $c$, the equality (1.2) is established with $\delta$ being replaced by $c$. The existence of eigenvalues, associated with eigenvectors in $\ell_{p}$, of subdivision operators is investigated in Section 3. For the scalar case, i.e., $r=1$, we show that $S_{a}$ has no eigenvalue in the open disc $\left\{z:|z|<\rho_{p}\left(S_{a}\right)\right\}$ in some cases. Moreover, if the subdivision operator generates a convergent subdivision scheme, we show that $S_{a}$ has no eigenvector in $\ell_{p}(\mathbb{Z})$.

Although we present all results only in the univariate case, our methods apply for the multivariate case except for partial results of Theorem 3.1, Corollary 3.2 and Theorem 3.7.

At the end of this section we cite the notion of $p$-joint spectral radii for the convenience of the readers. The uniform joint spectral radius was introduced in [12] and was employed to investigate the regularity of the refinable function in [4]. The mean joint spectral radius was introduced in [13]. For $1<p<\infty$, the $p$-joint spectral radius was introduced in [7] and applied to the study of $L_{p}$ convergence of cascade algorithms. We recall from [7] the definition of $p$-joint spectral radius.

If $V$ is a finite-dimensional space, let $\mathcal{B}(V)$ denote the collection of linear operators on $V$. Suppose that $\mathcal{A} \subseteq \mathcal{B}(V)$ is a finite set. For a positive integer $n$ we denote by $\mathcal{A}^{n}$ the Cartesian power of $\mathcal{A}$ :

$$
\mathcal{A}^{n}=\left\{\left(A_{1}, \ldots, A_{n}\right): A_{1}, \ldots, A_{n} \in \mathcal{A}\right\} .
$$

Suppose that $\mathcal{B}(V)$ is equipped with a norm $\|\cdot\|$, not necessarily the operator norm. For $1 \leq p<\infty$, let

$$
\left\|\mathcal{A}^{n}\right\|_{p}=\left(\sum_{\left(A_{1}, \ldots, A_{n}\right) \in \mathcal{A}^{n}}\left\|A_{1} \cdots A_{n}\right\|^{p}\right)^{1 / p},
$$

and for $p=\infty$, define $\left\|\mathcal{A}^{n}\right\|_{\infty}=\max \left\{\left\|A_{1} \cdots A_{n}\right\|:\left(A_{1}, \ldots, A_{n}\right) \in \mathcal{A}^{n}\right\}$. The $p$-joint spectral radius of $\mathcal{A}$, for $1 \leq p \leq \infty$, is defined to be

$$
\rho_{p}(\mathcal{A}):=\lim _{n \rightarrow \infty}\left\|\mathcal{A}^{n}\right\|_{p}^{\frac{1}{n}} .
$$

This limit indeed exists and does not depend on the choice of norm on $\mathcal{B}(V)$ ([7]). In particular, if we choose the norm such that $\|A\|=\left\|A^{*}\right\|$ for any $A \in \mathcal{B}(V)$ with 
$A^{*}$ being its adjoint, then $\left\|\mathcal{A}^{n}\right\|_{p}=\left\|\mathcal{A}^{* n}\right\|$, where $\mathcal{A}^{*}:=\left\{A^{*}: A \in \mathcal{A}\right\}$. It follows that

$$
\rho_{p}(\mathcal{A})=\rho_{p}\left(\mathcal{A}^{*}\right) .
$$

\section{$\S 2$. Spectral Radil of Subdivision operators}

In this section we establish some formulas for spectral radii of subdivision operators. In particular, we generalize the formula (2.5) considerably.

In what follows, for $\varepsilon=0,1$, let $A_{\varepsilon} \in \mathcal{B}\left(\mathbb{C}^{r N \times l}\right)$ be defined as follows:

$$
A_{\varepsilon} c(\alpha)=\sum_{\beta=0}^{N-1} a(\varepsilon+2 \alpha-\beta) c(\beta) \quad \forall \alpha=0,1, \ldots, N-1
$$

for

$$
c=\left(\begin{array}{c}
c(0) \\
c(1) \\
\vdots \\
c(N-1)
\end{array}\right) \in \mathbb{C}^{r N \times l},
$$

where $c(\beta) \in \mathbb{C}^{r \times r} \forall \beta=0,1, \ldots, N-1$. Clearly, the adjoint $A_{\varepsilon}^{*} \in \mathcal{B}\left(\mathbb{C}^{l \times r N}\right)$ is given by

$$
A_{\varepsilon}^{*} c(\beta)=\sum_{\alpha=0}^{N-1} c(\alpha) a(\varepsilon+2 \alpha-\beta) \quad \forall \beta=0,1, \ldots, N-1,
$$

for any $c=(c(0), \ldots, c(N-1)) \in \mathbb{C}^{l \times r N}$. In [3] we pay our attention to $\mathcal{A}:=$ $\left\{A_{0}, A_{1}\right\}$ for deducing the convergence of cascade algorithms in Sobolev spaces to that in $L_{p}$ space, while we are concerned with $\mathcal{A}^{*}$ for spectra of subdivision operators in this paper.

Let $\alpha \in \mathbb{Z}$. For any nonnegative integer $n$, there are uniquely $\varepsilon_{j} \in\{0,1\}, 1 \leq$ $j \leq n$, and $\gamma \in \mathbb{Z}$ such that $\alpha=2^{n} \gamma+2^{n-1} \varepsilon_{n}+\cdots+\varepsilon_{1}$. For any $c \in(\ell(\mathbb{Z}))^{l \times r}$, it is known (see [7]) that for $k \in K_{N}:=\{0,1, \ldots, N-1\}$,

$$
S_{a}^{n} c(\alpha-k)=A_{\varepsilon_{1}}^{*} \cdots A_{\varepsilon_{n}}^{*}{ }^{\gamma} c(k) \quad \forall \alpha \in \mathbb{Z}, k \in K_{N},
$$

where ${ }^{\gamma} c \in \mathbb{C}^{l \times r N}$, for $c=(c(\alpha))_{\alpha \in \mathbb{Z}} \in(\ell(\mathbb{Z}))^{l \times r}$ and for any $\gamma \in \mathbb{Z}$, is the restriction of $c(\gamma-\cdot)$ on $K_{N}$, i.e.,

$$
{ }^{\gamma} c=(c(\gamma), c(\gamma-1), \ldots, c(\gamma-N+1)) \in \mathbb{C}^{l \times r N} .
$$

Summing over $\alpha \in \mathbb{Z}$ and $k \in K_{N}$ gives

$$
N\left\|S_{a}^{n} c\right\|_{p}^{p}=\sum_{\varepsilon_{n}, \ldots, \varepsilon_{1}} \sum_{\gamma \in \mathbb{Z}}\left\|A_{\varepsilon_{1}}^{*} \cdots A_{\varepsilon_{n}}^{*} \gamma\right\|^{p} .
$$

For any $c \in(\ell(\mathbb{Z}))^{l \times r}$, let $U(c) \subseteq \mathbb{C}^{l \times r N}$ be the subspace spanned by all vectors

$$
A_{\eta_{1}}^{*} \cdots A_{\eta_{j}}^{*} c, \quad \gamma \in \mathbb{Z}, \eta_{j}=0,1, \quad j=0,1,2, \ldots
$$

It is the smallest invariant subspace of both $A_{0}^{*}$ and $A_{1}^{*}$, and is called the invariant subspace of both $A_{0}^{*}$ and $A_{1}^{*}$ generated by $c$.

Theorem 2.1. Suppose that $c \in\left(\ell_{p}(\mathbb{Z})\right)^{l \times r}$. Then

$$
\lim _{n \rightarrow \infty}|| S_{a}^{n} c||_{p}^{\frac{1}{n}}=\rho_{p}\left(\left\{\left.A_{0}^{*}\right|_{U(c)},\left.A_{1}^{*}\right|_{U(c)}\right\}\right)=\rho_{p}\left(\left.S_{a}\right|_{L(c)}\right),
$$

where $L(c)$ is the smallest closed invariant subspace of $S_{a}$ containing $c$. 
Proof. Note that $U(c) \subseteq \mathbb{C}^{l \times r N}$ is of finite dimension. There is a nonnegative $j_{0}$ such that $U(c)$ is spanned by the set

$$
\left\{A_{\eta_{1}}^{*} \cdots A_{\eta_{j}}^{*} c: \gamma \in \mathbb{Z}, \eta_{j}=0,1, j=0,1, \ldots, j_{0}\right\}
$$

Consequently,

$$
\|A\|:=\left(\sum_{\gamma \in \mathbb{Z}} \sum_{\eta_{1}, \cdots \eta_{j_{0}}}\left\|A A_{\eta_{1}}^{*} \cdots A_{\eta_{j_{0}}}^{*} \gamma^{\gamma}\right\|^{p}\right)^{1 / p}, \quad A \in \mathcal{B}(U(c)),
$$

defines a norm on the set $\mathcal{B}(U(c))$. We note that the series in the right-hand side of the last equality converges for any $c \in\left(\ell_{p}(\mathbb{Z})\right)^{l \times r}$. Since $\mathcal{B}(U(c))$ is of finite dimension, there is a constant $\kappa>0$ satisfying

$$
\|A u\| \leq \kappa\|A\|\|u\| \quad \forall u \in U(c), A \in \mathcal{B}(U(c)) .
$$

Let $\mathcal{A}=\left\{\left.A_{0}^{*}\right|_{U(c)},\left.A_{1}^{*}\right|_{U(c)}\right\} \subseteq \mathcal{B}(U(c))$. Recall the definition of $\left\|\mathcal{A}^{n}\right\|_{p}$ with norm (2.6) on $\mathcal{B}(U(c))$. It follows from (2.4) that $N\left\|S_{a}^{n+j_{0}} c\right\|_{p}^{p}=\left\|\mathcal{A}^{n}\right\|_{p}^{p}$. Therefore, the first equality in (2.5) is true by (1.3).

Suppose $d \in L(c)$. It is easily seen that ${ }^{\gamma} d \in U(c) \forall \gamma \in \mathbb{Z}$. The inequality (2.7) gives

$$
\left(\sum_{\varepsilon_{n}, \ldots, \varepsilon_{1}} \sum_{\gamma \in \mathbb{Z}}\left\|A_{\varepsilon_{1}}^{*} \cdots A_{\varepsilon_{n}}^{*}{ }^{\gamma} d\right\|^{p}\right)^{1 / p} \leq \kappa\left\|\mathcal{A}^{n}\right\|_{p}\left(\sum_{\gamma \in \mathbb{Z}}\|\|^{\gamma} d \|^{p}\right)^{1 / p} .
$$

Note that $\sum_{\gamma \in \mathbb{Z}}\left\|{ }^{\gamma} d\right\|^{p}=N\|d\|_{p}^{p}$. We obtain by $(2.7),\left\|S_{a}^{n} d\right\|_{p} \leq \kappa\left\|\mathcal{A}^{n}\right\|_{p}\|d\|_{p}, n=$ $1,2, \ldots$ This implies that $\left\|\left(\left.S_{a}\right|_{L(c)}\right)^{n}\right\| \leq \kappa\left\|\mathcal{A}^{n}\right\|_{p}, n=1,2, \ldots$ Therefore, $\rho_{p}\left(\left.S_{a}\right|_{L(c)}\right) \leq \rho_{p}(\mathcal{A})$. The reverse inequality holds by the fact that $\lim _{n \rightarrow \infty}\left\|S_{a}^{n} c\right\|_{p}^{1 / n}$ $\leq \rho_{p}\left(\left.S_{a}\right|_{L(c)}\right)$ and the first equality in (2.5). This establishes the second equality in (2.5), thereby completing the proof of the theorem.

Corollary 2.2. Suppose that $S \subseteq\left(\ell_{p}(\mathbb{Z})\right)^{l \times r}$ is an invariant subspace of $S_{a}$. Then

$$
\rho_{p}\left(\left.S_{a}\right|_{S}\right)=\max _{c \in S} \lim _{n \rightarrow \infty}\left\|S_{a}^{n} c\right\|_{p}^{\frac{1}{n}} .
$$

Proof. Since $S=\bigcup_{c \in S} L(c)$, we get $\rho_{p}\left(\left.S_{a}\right|_{S}\right)=\sup _{c \in S} \rho_{p}\left(\left.S_{a}\right|_{L(c)}\right)$. Therefore Theorem 2.1 gives

$$
\rho_{p}\left(\left.S_{a}\right|_{S}\right)=\sup _{c \in S} \rho_{p}\left(\left\{\left.A_{0}^{*}\right|_{U(c)},\left.A_{1}^{*}\right|_{U(c)}\right\}\right)=\rho_{p}\left(\left\{\left.A_{0}^{*}\right|_{U(S)},\left.A_{1}^{*}\right|_{U(S)}\right\}\right),
$$

where $U(S):=\bigcup_{c \in S} U(c)$. Note that $U(S)$ is also a finite-dimensional subspace and, thus, may be decomposed as a finite sum $U(S)=U\left(c^{1}\right)+\cdots+U\left(c^{k}\right)$ for some $c^{j} \in S, 1 \leq j \leq k$, where $k$ is a positive integer. By the definition of joint spectral radius, it follows that

$$
\rho_{p}\left(\left\{\left.A_{0}^{*}\right|_{U(S)},\left.A_{1}^{*}\right|_{U(S)}\right\}\right)=\max _{1 \leq j \leq k} \rho_{p}\left(\left\{\left.A_{0}^{*}\right|_{U\left(c^{j}\right)},\left.A_{1}^{*}\right|_{U\left(c^{j}\right)}\right\}\right) .
$$

The proof is complete by the first equality in (2.5).

In what follows we want to give a simple condition on $c$ that ensures that

$$
\rho_{p}\left(\left\{A_{0}^{*}, A_{0}^{*}\right\}\right)=\rho_{p}\left(\left\{\left.A_{0}^{*}\right|_{U(c)},\left.A_{0}^{*}\right|_{U(c)}\right\}\right) .
$$

To this end, we consider the joint spectral radii concerning the following subspace:

$$
U(* c):=\bigcup\left\{U(\lambda): \lambda=\eta * c, \eta \in\left(\ell_{0}(\mathbb{Z})\right)^{1 \times l}\right\} \subseteq \mathbb{C}^{1 \times r N} .
$$


Theorem 2.3. For any $c \in\left(\ell_{p}(\mathbb{Z})\right)^{l \times r}$, we have

$$
\lim _{n \rightarrow \infty} \| S_{a}^{n} c||_{p}^{\frac{1}{n}}=\max _{\lambda \in U(* c)} \rho_{p}\left(\left\{\left.A_{0}^{*}\right|_{U(\lambda)},\left.A_{1}^{*}\right|_{U(\lambda)}\right\}\right)=\rho_{p}\left(\left\{\left.A_{0}^{*}\right|_{U(* c)},\left.A_{1}\right|_{U(* c)}\right\}\right) .
$$

Proof. The second equality in (2.10) follows in the same way as (2.8). We prove now the first equality in $(2.10)$.

If $\lambda \in U(* c)$, there is an $\eta \in\left(\ell_{0}(\mathbb{Z})\right)^{1 \times l}$ such that $\lambda=\eta * c$. Appealing to (2.3) we have

$$
S_{a}^{n} \lambda(\alpha-k)=\sum_{\gamma^{\prime} \in \mathbb{Z}} \eta\left(\gamma^{\prime}\right) A_{\varepsilon_{1}}^{*} \cdots A_{\varepsilon_{n}}^{*} c^{\gamma-\gamma^{\prime}}(k), \quad 0 \leq k \leq N-1,
$$

where $\gamma \in \mathbb{Z}, \varepsilon_{1}, \ldots$ and $\varepsilon_{n}$ are determined by $\alpha$ in the same way as (2.3). Therefore $\left\|S_{a}^{n} \lambda\right\|_{p} \leq \kappa|| S_{a}^{n} c \mid\left\|_{p}\right\| \eta \|_{1}$ for some constant $\kappa$ independent of $n$ and $\lambda$.

On the other hand, for $j=1, \ldots, l$, with a special choice of $\eta^{j} \in\left(\ell_{0}(\mathbb{Z})\right)^{1 \times l}$ and $\lambda^{j}=\eta^{j} * c, S_{a}^{n} \lambda^{j}(\alpha)$ specifies the $j$ th row of $S_{a}^{n} c(\alpha), \alpha \in \mathbb{Z}$. Thus,

$$
\left\|S_{a}^{n} c\right\|_{p} \leq \max _{j=1, \ldots, l}\left\|S_{a}^{n} \lambda^{j}\right\|_{p} \leq \kappa^{\prime}\left\|S_{a}^{n} c\right\|_{p} .
$$

Taking the $1 / n$ power and letting $n \rightarrow \infty$ in the above inequalities we obtain by Theorem 2.1,

$$
\max _{j=1, \ldots, l n \rightarrow \infty} \lim _{n \rightarrow \infty}\left\|S_{a}^{n} \lambda^{j}\right\|_{p}^{\frac{1}{n}}=\rho_{p}\left(\left\{\left.A_{0}^{*}\right|_{U(c)},\left.A_{1}^{*}\right|_{U(c)}\right\}\right) .
$$

Applying Theorem 2.1 to the left-hand side of the above equality for $c=\lambda^{j}, j=$ $1, \ldots, l$, we obtain the first equality in $(2.10)$. The proof is complete.

For any $u \in \mathbb{C}^{l \times N}$, we denote by $u_{j} \in \mathbb{C}^{1 \times r N}$ the $j$ th row of $u$. If $U$ is a subset of $\mathbb{C}^{l \times r N}$, let

$$
\mathcal{R}(U):=\operatorname{span}\left\{u_{j}: u \in U, 1 \leq j \leq l\right\} \subseteq \mathbb{C}^{1 \times r N} .
$$

It is easily seen that $\mathcal{R}(U)$ is an invariant subspace of both $A_{0}^{*}$ and $A_{1}^{*}$ if $U$ is. Moreover, $\rho_{p}\left(\left\{\left.A_{0}^{*}\right|_{U},\left.A_{1}^{*}\right|_{U}\right\}\right)=\rho_{p}\left(\left\{\left.A_{0}^{*}\right|_{\mathcal{R}(U)},\left.A_{1}^{*}\right|_{\mathcal{R}(U)}\right\}\right)$ provided that $U$ is invariant under both $A_{0}^{*}$ and $A_{1}^{*}$. In particular, for any $c \in\left(\ell_{p}(\mathbb{Z})\right)^{l \times r}$ we have

$$
\rho_{p}\left(\left\{\left.A_{0}^{*}\right|_{U(c)},\left.A_{1}^{*}\right|_{U(c)}\right\}\right)=\rho_{p}\left(\left\{\left.A_{0}^{*}\right|_{\mathcal{R}(U(c))},\left.A_{1}^{*}\right|_{\mathcal{R}(U(c))}\right) .\right.
$$

Although the following auxiliary lemma is undoubtedly well known, we would like to present a proof for convenience. Recall that $K_{N}=\{0,1, \ldots, N-1\}$. Let $\left(\ell\left(K_{N}\right)\right)^{r \times 1}$ denote the subspace consisting of $c \in(\ell(\mathbb{Z}))^{r \times 1}$ with $\operatorname{supp} c \in K_{N}$.

Lemma 2.4. Let $c \in\left(\ell_{p}\left(\mathbb{Z}^{s}\right)\right)^{l \times r}$ satisfy

$$
c * t \neq 0 \quad \forall t \in\left(\ell\left(K_{N}\right)\right)^{r \times 1} \backslash\{0\} .
$$

Then

$$
\mathcal{R}\left(\left\{{ }^{\gamma} c: \gamma \in \mathbb{Z}\right\}\right)=\mathbb{C}^{1 \times r N} .
$$

Consequently, in this case,

$$
\|A\|:=\left(\sum_{\gamma \in \mathbb{Z}}\left\|A^{\gamma} c\right\|^{p}\right)^{1 / p}
$$

is a norm on $\mathcal{B}\left(\mathbb{C}^{1 \times r N}\right)$. 
Proof. The formula (2.14) follows easily from (2.14). The proof of (2.14) is elementary. Note that $\mathcal{R}\left(\left\{{ }^{\gamma} c: \gamma \in \mathbb{Z}\right\}\right) \subseteq \mathbb{C}^{1 \times r N}$ is spanned by the vectors

$$
{ }^{\gamma} c_{j}:=\left(c_{j}(\gamma), c_{j}(\gamma-1), \ldots, c_{j}(\gamma-N+1)\right), \quad \gamma \in \mathbb{Z}, 1 \leq j \leq r,
$$

where $c_{j}(\alpha) \in \mathbb{C}^{1 \times r}$ is the $j$ th row of $c(\alpha), \alpha \in \mathbb{Z}$. Assume to the contrary that (2.14) is not true. There would be a nonzero column vector $t=(t(0), \ldots, t(N-1))^{\mathrm{T}} \in$ $\mathbb{C}^{r N \times 1}$, with $t(\alpha) \in \mathbb{C}^{r \times 1}$, satisfying

$$
\sum_{\alpha=0}^{N-1} c_{j}(\gamma-\alpha) t(\alpha)=0 \quad \forall \gamma \in \mathbb{Z}, 1 \leq j \leq r .
$$

We then extend $t$ to a vector in $\left(\ell\left(K_{N}\right)\right)^{r \times 1} \backslash\{0\}$, denoted by $t$ again, by setting $t(\alpha)=0$ for any $\alpha \notin K_{N}$, so that (2.12) holds. This contradicts our assumptions. The proof is complete.

Remark 2.5. We point out that (2.12) is satisfied for $c \in\left(\ell_{p}(\mathbb{Z})\right)^{l \times r} \backslash\{0\}$ in the following cases:

(1) $l=r=1$ and $1 \leq p \leq 2$;

(2) $l=r=1$ and the univariate case.

The following result extends (1.2) to a much more general setting.

Theorem 2.6. Assume that $c \in\left(\ell_{p}(\mathbb{Z})\right)^{l \times r}$ satisfies the condition (2.12). Then

$$
\rho_{p}\left(S_{a}\right)=\rho_{p}\left(\left\{A_{0}, A_{1}\right\}\right)=\lim _{n \rightarrow \infty}\left\|S_{a}^{n} c\right\|_{p}^{\frac{1}{n}} .
$$

Proof. The proof of the first equality is the same as [7]. To establish the second equality, by (1.4) and Theorem 2.1 , it suffices to prove $\rho_{p}\left(\left\{\left.A_{0}^{*}\right|_{U(c)},\left.A_{1}^{*}\right|_{U(c)}\right\}\right)=$ $\rho_{p}\left(\left\{A_{0}^{*}, A_{1}^{*}\right\}\right)$. We note first that $\rho_{p}\left(\left\{\left.A_{0}^{*}\right|_{U(c)},\left.A_{1}^{*}\right|_{U(c)}\right\}\right) \leq \rho_{p}\left(\left\{A_{0}^{*}, A_{1}^{*}\right\}\right)$.

Since $\mathcal{R}\left(\left\{{ }^{\gamma} c: \gamma \in \mathbb{Z}\right\}\right) \subseteq \mathcal{R}(U(c))$, we have

$$
\rho_{p}\left(\left\{\left.A_{0}^{*}\right|_{\mathcal{R}\left(\left\{\gamma_{c: \gamma \in \mathbb{Z}\}}\right)\right.},\left.A_{1}^{*}\right|_{\mathcal{R}\left(\left\{\gamma_{c: \gamma} \in \mathbb{Z}\right\}\right)}\right\}\right) \leq \rho_{p}\left(\left\{\left.A_{0}^{*}\right|_{\mathcal{R}(U(c))},\left.A_{1}^{*}\right|_{\mathcal{R}(U(c))}\right\}\right) .
$$

By $(2.11), \rho_{p}\left(\left\{\left.A_{0}^{*}\right|_{\mathcal{R}\left(\left\{\gamma_{c}: \gamma \in \mathbb{Z}\right\}\right)},\left.A_{1}^{*}\right|_{\mathcal{R}\left(\left\{\gamma_{c}: \gamma \in \mathbb{Z}\right\}\right)}\right\}\right) \leq \rho_{p}\left(\left\{\left.A_{0}^{*}\right|_{U(c)},\left.A_{1}^{*}\right|_{U(c)}\right\}\right)$.

On the other hand, by the assumptions we know from Lemma 2.4 that $\mathcal{R}\left(\left\{{ }^{\gamma} c\right.\right.$ : $\gamma \in \mathbb{Z}\}=\mathbb{C}^{1 \times r N}$. Moreover, it is easily seen that $\rho_{p}\left\{A_{0}^{*}, A_{1}^{*}\right\}$ is independent of $l$, i.e., $\rho_{p}\left\{A_{0}^{*}, A_{1}^{*}\right\}=\rho_{p}\left\{\left.A_{0}^{*}\right|_{\mathbb{C}^{1 \times r N}},\left.A_{1}^{*}\right|_{\mathbb{C}^{1 \times r N}}\right\}$. It follows that

$$
\rho_{p}\left(\left\{A_{0}^{*}, A_{1}^{*}\right\}\right)=\rho_{p}\left(\left\{\left.A_{0}^{*}\right|_{\mathcal{R}\left(\left\{\gamma_{c: \gamma} \in \mathbb{Z}\right\}\right)},\left.A_{1}^{*}\right|_{\mathcal{R}\left(\left\{{ }^{\gamma} c: \gamma \in \mathbb{Z}\right\}\right)}\right\}\right) .
$$

The proof is complete.

With the help of Theorem 2.6, we may calculate the spectral radius of $S_{a}$ in terms of the moduli of its eigenvalues.

Theorem 2.7. Let $c^{j} \in\left(\ell_{p}\left(\mathbb{Z}^{s}\right)\right)^{1 \times r}$ be an eigenvector of $S_{a}$ with eigenvalues $\sigma_{j}, j=1, \ldots, l$, respectively. Suppose that $c:=\left(c^{1}, \ldots, c^{l}\right)^{\mathrm{T}} \in\left(\ell_{p}(\mathbb{Z})\right)^{l \times r}$ satisfies the condition (2.12). Then $\rho_{p}\left(S_{a}\right)=\max \left\{\left|\sigma_{1}\right|, \ldots,\left|\sigma_{l}\right|\right\}$.

Proof. Since $S_{a}^{n} c^{j}=\sigma_{j}^{n} c^{j}$ and $c^{j} \neq 0,1 \leq j \leq l$, we have $\lim _{n \rightarrow \infty}\left\|S_{a}^{n} c\right\|_{p}^{\frac{1}{n}}=$ $\max \left\{\left|\sigma_{1}\right|, \ldots,\left|\sigma_{l}\right|\right\}$. Therefore the proof is complete by Theorem 2.6. 


\section{§3. EIgENVECTORS OF SUBDivision OPERATORS}

In this section we discuss the eigenvectors of a subdivision operator $S_{a}$. We prove that $S_{a}$ has no eigenvalue in the open disc $\left\{z:|z|<\rho_{p}\left(S_{a}\right)\right\}$ in two cases as listed in Remark 2.5. Moreover, we have these cases, it is shown that, if $S_{a}$ generates a convergent subdivision scheme, it has no an eigenvector in $\ell_{p}$.

Theorem 3.1. Suppose that either $l=r=1$ and $1 \leq p \leq 2$ or $l=r=1$ and we have the univariate case. Then $S_{a}$ has no eigenvector in $\ell_{p}(\mathbb{Z})$ corresponding to an eigenvalue in the disc

$$
\left\{z \in \mathbb{C}:|z|<\rho_{p}\left(S_{a}\right)\right\} .
$$

Proof. It follows immediately from Remark 2.5 and Theorem 2.7 and the discussion at the end of last section.

Under the assumption $\widehat{a}(\omega) \neq 0 \forall \omega \in \mathbb{R}$, Theorem 3.1 was established for $p=2$ in [6] by a different method, which, on the other hand, applies to the case when $a$ is not finitely supported.

By Theorem 2 of [15] and Theorem 3.1 we have

Corollary 3.2. Under the hypotheses of Theorem 3.1, any point in the disc (3.1) lies in the residual spectrum of $S_{a}$.

In the case $r>1$, we can construct an example in which $S_{a}$ has an eigenvalue $\sigma$ with $|\sigma|<\rho_{\infty}\left(S_{a}\right)$. First we choose a sequence $a_{1} \in \ell_{0}(\mathbb{Z})$ satisfying $\rho_{\infty}\left(S_{a_{1}}\right)>1$. Let $a_{2} \in \ell_{0}(\mathbb{Z})$ be given by $a_{2}(0)=a_{2}(1)=1$ and $a_{2}(\alpha)=0 \forall \alpha \notin\{0,1\}$. Clearly, $e:=(\ldots, 1,1,1, \ldots)$ is an eigenvector of $S_{a_{2}}$ with eigenvalue 1 . Then we define $a \in\left(\ell_{0}(\mathbb{Z})\right)^{2 \times 2}$ by $a(\alpha)=\operatorname{diag}\left(a_{1}(\alpha), a_{2}(\alpha)\right) \forall \alpha \in \mathbb{Z}$. It is easy to check that

$$
1=\rho_{\infty}\left(S_{a_{2}}\right)<\rho_{\infty}\left(S_{a_{1}}\right)=\rho_{\infty}\left(S_{a}\right) .
$$

On the other hand, $(0, e)$ is an eigenvector of $S_{a}$ with eigenvalue 1 , where 0 stands for the zero element of $\ell(\mathbb{Z})$.

We now restrict ourselves to the subdivision operators that generate convergent subdivision schemes.

Lemma 3.3. If the subdivision scheme associated with a converges in $L_{p}$ for some $p$, then the spectral radius of $S_{a}$ satisfies $\rho_{p}\left(S_{a}\right)=2^{1 / p}$.

Proof. We note first that, for any $g \in\left(W_{p}(\mathbb{R})\right)^{l \times r}$ and $\mu_{p}^{n}(g)$ given in Section 1 , it follows that $\lim _{n \rightarrow \infty} 2^{-n / p}\left\|\mu_{p}^{n}(g)\right\|_{p}=\|g\|_{p}$. This together with the equality (1.1) yields that, for any $c \in\left(\ell_{p}(\mathbb{Z})\right)^{l \times r}, \lim _{n \rightarrow \infty} 2^{-n / p}\left\|S_{a}^{n} c\right\|_{p}=\left\|f_{c}\right\|_{p}$, where $f_{c}$ is given by (1.1). Consequently, for any $c \in\left(\ell_{p}(\mathbb{Z})\right)^{l \times r}$,

$$
\lim _{n \rightarrow \infty}\left\|S_{a}^{n} c\right\|_{p}^{1 / n}= \begin{cases}2^{1 / p}, & f_{c} \neq 0 \\ 0, & f_{c}=0\end{cases}
$$

But, the notion of convergence of the subdivision scheme implies that there is at least a $c \in\left(\ell_{p}(\mathbb{Z})\right)^{l \times r}$ such that $f_{c} \neq 0$. Therefore, $\rho_{p}\left(S_{a}\right) \geq 2^{1 / p}$.

In particular, we may choose a $c \in\left(\ell_{p}(\mathbb{Z})\right)^{l \times r}$ satisfying (2.12). Then Theorem 2.6 gives $\lim _{n \rightarrow \infty}\left\|S_{a}^{n} c\right\|_{p}^{1 / n}=\rho_{p}\left(S_{a}\right)$. As is known, $\lim _{n \rightarrow \infty}\left\|S_{a}^{n} c\right\|_{p}^{1 / n}=0$ or $2^{1 / p}$. It follows that $\rho_{p}\left(S_{a}\right) \leq 2^{1 / p}$. Therefore, the result of the lemma follows. 
Lemma 3.4. Suppose $c^{1} \in\left(\ell_{p}(\mathbb{Z})\right)^{1 \times r}$ satisfies

$$
{ }^{\alpha} c^{1} A_{\varepsilon}={ }^{\alpha} c^{1} \quad \forall \alpha \in \mathbb{Z}, \quad \varepsilon=0,1 .
$$

Then the following conclusions are true.

(1) For any constant $\sigma \neq 1, c^{1}$ is not an eigenvector of $S_{a}$ with eigenvalue $\sigma$.

(2) If $c^{1}$ is an eigenvector of $S_{a}$ with eigenvalue $\sigma$, then $p=\infty$ and

$$
c^{1}(\alpha)=c^{1}(0) \quad \forall \alpha \in \mathbb{Z} .
$$

Proof. We establish (1) first. Suppose to the contrary that $c^{1}$ is an eigenvector of $S_{a}$ with eigenvalue $\sigma \neq 1$. This means that $S_{a} c^{1}=\sigma c^{1}$ and $c^{1} \neq 0$. But we are able to prove $c^{1}=0$ as follows.

In fact, it follows from (2.3) and (3.2) that

$$
{ }^{\alpha} c=\sigma\left({ }^{2 \alpha+\varepsilon} c\right) \quad \forall \alpha \in \mathbb{Z}, \quad \varepsilon=0,1 .
$$

If $\sigma=0$, it follows from the above equality that $c^{1}(\alpha)=\sigma c^{1}(2 \alpha+\varepsilon)=0, \alpha \in \mathbb{Z}$.

If $\sigma \neq 0$, setting $\alpha=\varepsilon=0$ in (3.4) we get ${ }^{0} c=0$. Recursively we may deduce that ${ }^{\alpha} c=0$ for any $\alpha \geq 0$. For example, ${ }^{1} c=0$ is true by letting $\alpha=0$ and $\varepsilon=1$ in (3.4).

For $\alpha<0$, the proof for ${ }^{\alpha} c=0$ is similar as above. We only note that ${ }^{-1} c=0$ by setting $\alpha=-1$ and $\varepsilon=1$ in (3.4). We have $c^{1}=0$, as desired. This proves (1).

Suppose now that $c^{1}$ is an eigenvector of $S_{a}$ with eigenvalue 1 . As in the proof of (1), we may deduce that $c^{1}$ satisfies (3.3). Note $c^{1} \in\left(\ell_{p}(\mathbb{Z})\right)^{1 \times r}$. We conclude that $p=\infty$ and, therefore, complete the proof.

Theorem 3.5. Suppose that the subdivision scheme associated with a converges in $L_{p}$ for some $1 \leq p \leq \infty$. If $\sigma$ is an eigenvalue of $S_{a}$ on $\left(\ell_{p}(\mathbb{Z})\right)^{1 \times r}$, then the following statements are true.

(1) If $1 \leq p<\infty$, then $|\sigma|<2^{1 / p}$.

(2) If $p=\infty$, then either $|\sigma|<1$ or $\sigma=1$. Furthermore, if $\sigma=1$, the corresponding eigenvector $c^{1}$ satisfies (3.3).

Proof. Suppose that $1 \leq p<\infty$. We claim that if the subdivision scheme associated with $a$ converges in $L_{p}$ then, for any $d \in\left(\ell_{p}(\mathbb{Z})\right)^{1 \times r}$, there is a positive constant $\tau<1$, dependent on $d$, satisfying

$$
2^{-n} \sum_{\gamma \in \mathbb{Z}} \sum_{\varepsilon_{n}, \ldots, \varepsilon_{1}}\left\|\left.\right|^{\gamma} d A_{\varepsilon_{n}} \cdots A_{\varepsilon_{1}}\left(A_{\varepsilon}-I\right)\right\|^{p} \leq \tau^{n} \quad \forall \varepsilon=0,1, \quad n=1,2, \ldots
$$

where $I$ is the identity matrix of the appropriate size.

Recall that, for any $d \in\left(\ell_{p}(\mathbb{Z})\right)^{1 \times r}$, the function $f_{d}$ is given by (1.1). Since $f_{d} \in\left(L_{p}(\mathbb{R})\right)^{1 \times r}$, it is easy to check that

$$
\lim _{n \rightarrow \infty} 2^{-n} \sum_{k \in K_{N}} \sum_{\alpha \in \mathbb{Z}}\left\|\mu_{p}^{n+1}\left(f_{d}\right)(2 \alpha+\varepsilon-k)-\mu_{p}^{n}\left(f_{d}\right)(\alpha-k)\right\|^{p}=0, \quad \varepsilon=0,1 .
$$

It follows from (1.1) that, for $d \in\left(\ell_{p}(\mathbb{Z})\right)^{1 \times r}$,

$$
\lim _{n \rightarrow \infty} 2^{-n} \sum_{k \in K_{N}} \sum_{\alpha \in \mathbb{Z}}\left\|S_{a}^{n+1} d(2 \alpha+\varepsilon-k)-S_{a}^{n} d(\alpha-k)\right\|^{p}=0, \quad \varepsilon=0,1 .
$$

Substituting (2.3) into the above equality we obtain for $d \in\left(\ell_{p}(\mathbb{Z})\right)^{1 \times r}$ that

$$
\lim _{n \rightarrow \infty} 2^{-n} \sum_{\gamma \in \mathbb{Z}} \sum_{\varepsilon_{n}, \ldots, \varepsilon_{1}}\left\|\left.\right|^{\gamma} d A_{\varepsilon_{n}} \cdots A_{\varepsilon_{1}}\left(A_{\varepsilon}-I\right)\right\|^{p}=0, \quad \varepsilon=0,1 .
$$


Since $c^{1 \times r N}$ is of finite dimension, it is not difficult to derive (3.5) from the last relation (see, e.g., the proof of Theorem 2.6 of [9]). Therefore (3.5) is true, as claimed.

Suppose that $c^{1} \in\left(\ell_{p}(\mathbb{Z})\right)^{1 \times r}$ is an eigenvector of $S_{a}$ with eigenvalue $\sigma$. Let $\alpha=2^{n} \gamma+2^{n-1} \varepsilon_{n}+\cdots+\varepsilon_{1}$. Then

$$
{ }^{\gamma} c A_{\varepsilon_{n}} \ldots A_{\varepsilon_{1}}\left(A_{\varepsilon}-I\right)=\sigma^{n}\left({ }^{\alpha} c^{1}\left(A_{\varepsilon}-I\right)\right), \quad \varepsilon=0,1 .
$$

Meanwhile, appealing to Lemma 3.4 we know that (3.2) is not true. Therefore, $\sum_{\alpha \in \mathbb{Z}}||^{\alpha} c^{1}\left(A_{\varepsilon}-I\right) \|^{p} \neq 0$ for some $\varepsilon$.

Setting $d=c^{1}$ in the left-hand side of (3.5) and taking (3.6) into account we obtain

$$
\begin{aligned}
& \lim _{n \rightarrow \infty}\left(2^{-n} \sum_{\gamma \in \mathbb{Z}} \sum_{\varepsilon_{n}, \ldots, \varepsilon_{1}}\left\|{ }^{\gamma} c A_{\varepsilon_{n}} \ldots A_{\varepsilon_{1}}\left(A_{\varepsilon}-I\right)\right\|^{p}\right)^{\frac{1}{n_{p}}} \\
= & \lim _{n \rightarrow \infty}\left(2^{-n}|\sigma|^{p n} \sum_{\alpha \in \mathbb{Z}} \|\left.\left.\right|^{\alpha} c^{1}\left(A_{\varepsilon}-I\right)\right|^{p}\right)^{\frac{1}{n_{p}}}=2^{-1 / p}|\sigma| .
\end{aligned}
$$

Therefore, the inequality (3.5) implies that $2^{-1 / p}|\sigma| \leq \tau<1$. This establishes (1).

The analogue of (3.5) for $p=\infty$ is, for a constant $\tau \in(0,1)$,

$$
2^{-n}\left\|^{\gamma} d A_{\varepsilon_{n}} \cdots A_{\varepsilon_{1}}\left(A_{\varepsilon}-I\right)\right\| \leq \tau^{n} \quad \forall \varepsilon_{n}, \ldots, \varepsilon_{1}, \varepsilon, \gamma \in \mathbb{Z}, \quad n=1,2, \ldots
$$

If $\sigma \neq 1$, we know that (3.2) is not true. Similar to the proof of (1) we can conclude that $|\sigma|<1$. The conclusion for $\sigma=1$ follows from Lemma 3.4. The proof is complete.

Corollary 3.6. Suppose that the subdivision scheme associated with a converges in $L_{p}$ for some $p \in[1, \infty)$. Then there are not eigenvectors $c^{j} \in\left(\ell_{p}(\mathbb{Z})\right)^{1 \times r}$ of $S_{a}, 1 \leq j \leq l$, such that $c:=\left(c^{1}, \ldots, c^{l}\right)^{\mathrm{T}} \in\left(\ell_{p}(\mathbb{Z})\right)^{l \times r}$ satisfies condition $(2.12)$.

Proof. Assume that $c^{j} \in\left(\ell_{p}(\mathbb{Z})\right)^{1 \times r}$ is an eigenvector of $S_{a}$ with eigenvalues $\sigma_{j}, 1 \leq$ $j \leq r$, respectively. Then by Theorem 3.3 we have $\left|\sigma_{j}\right|<2^{1 / p}$ for $1 \leq j \leq l$. Recall from Lemma 3.3 that $\rho_{p}\left(S_{a}\right)=2^{1 / p}$. This contradicts Theorem 2.7 if $c$ satisfies (2.12). The proof is complete.

Let $l=r=1$. As is known, for either (i) $1 \leq p \leq 2$ or (ii) $1 \leq p<\infty$ in the univariate case, any $c \in \ell_{p}(\mathbb{Z})$ satisfies (2.12). Combining Theorem 3.5 and Corollary 3.6 we have an interesting result as follows.

Theorem 3.7. Let $l=r=1$. Suppose that the subdivision scheme associated with a converges in $L_{p}$. Then for either (i) $1 \leq p \leq 2$ or (ii) $1 \leq p<\infty$ in the univariate case, $S_{a}$ has no eigenvector in $\ell_{p}(\mathbb{Z})$.

The author thanks Prof. Rong-Qing Jia and Dr. Ding-Xuan Zhou for their helpful comments.

\section{REFERENCES}

1. A. S. Cavaretta, W. Dahmen, and C. A. Micchelli, Stationary subdivision, Memoirs Amer. Math. Soc. 93 (1991), 1-186. MR 92h:65017

2. D. R. Chen, Algebraic properties of subdivision operators with matrix mask and their applications, J. Approx. Theory 97 (1999), 294-310. MR 2000a:41025

3. D. R. Chen, Construction of smooth refinable function vectors by cascade algorithms, SIAM J. Numer. Anal. 40 (2002), 1354-1368. 
4. I. Daubechies and J. Lagarias, Two-scale difference equations I. Existence and global regularity of solutions, SIAM J. Math. Anal. 22 (1991), 1388-1410. MR 92d:39001

5. T. N. T. Goodman, C. A. Micchelli, and J. D. Ward, Spectral radius formulas for subdivision operators, Recent Advances in Wavelet Analysis (L. L. Schumaker and G. Webb, eds.), Academic Press, Boston, MA, 1994, pp. 335-360. MR 94m:47076

6. Mark C. Ho, Spectra of slant Toeplitz operators with continuous symbols, Michigan Math. J. 44 (1997), 157-164. MR 98c:47034

7. R. Q. Jia, Subdivision schemes in $L_{p}$ spaces, Adv. Comput. Math. 3 (1995), 309-341. MR 96d:65028

8. R. Q. Jia, S. D. Riemenschneider, and D. X. Zhou, Approximation by multiple refinable functions, Canadian J. Math. 49 (1997), 944-962. MR 99f:39036

9. K. S. Lau and J. Wang, Characterization of $L^{p}$-solutions for two-scale dilation equations, SIAM J. Math. Anal. 26 (1995), 1018-1046. MR 96f:39004

10. C. A. Micchelli and T. Sauer, Regularity of multiwavelets, Adv. Comput. Math. 7 (1997), 455-545. MR 99d:42067

11. C. A. Micchelli and T. Sauer, On vector subdivision, Math. Z. 229 (1998), 621-674. MR 2000d: 42016

12. G.-C. Rota and G. Strang, A note on the joint spectral radius, Indag. Math. 22 (1960), 379-381. MR 26:5434

13. Y. Wang, Two-scale dilation equations and the mean spectral radius, Random and Computational Dynamics 4 (1996), 49-72. MR 96j:42023

14. D. X. Zhou, The p-norm joint spectral radius for even integers, Methods and Applications of Analysis 5 (1998), 39-54. MR 99e:42054

15. D. X. Zhou, Spectra of subdivision operators, Proc. Amer. Math. Soc. 129 (2001), 191-202. MR 2001h:47049

Department of Applied Mathematics, Beijing University of Aeronautics, AstronauTics, BeiJing 100083, China

Department of Mathematics, Hubei Institute for Nationalities, Enshi 445000, Hubei, CHINA

E-mail address: drchen@buaa.edu.cn 